\title{
Numerical study on the influence of initial anisotropy on optimal blank shape
}

\author{
R. Padmanabhan ${ }^{\mathrm{a}, *}$, M.C. Oliveira ${ }^{\mathrm{a}}$, A.J. Baptista ${ }^{\mathrm{a}}$, J.L. Alves ${ }^{\mathrm{b}}$, L.F. Menezes ${ }^{\mathrm{a}}$ \\ ${ }^{a}$ CEMUC, Department of Mechanical Engineering, University of Coimbra, Polo II, Pinhal de Marrocos, 3030-201 Coimbra, Portugal \\ ${ }^{\mathrm{b}}$ Department of Mechanical Engineering, University of Minho, Campus de Azurém, 4800-058 Guimarães, Portugal
}

\section{A R T I C L E I N F O}

\section{Article history:}

Received 17 December 2007

Received in revised form 14 May 2008

Accepted 20 July 2008

Available online 18 September 2008

\section{Keywords:}

Blank shape

Anisotropy

NURBS surface

Optimization

FEM

\begin{abstract}
A B S T R A C T
In this paper, the influence of the initial anisotropy in achieving an optimal blank shape is analysed using anisotropic mild steel (DC06) and nearly isotropic dual-phase steel (DP600) blanks for different tool geometries. An iterative blank shape optimization procedure is used to determine the optimal blank shapes for the geometries. The numerical method is based on an initial NURBS surface used to define the blank shape and the resulting flange geometry of the deformed part. Different rolling direction orientations were considered in the blanks for deep drawing to delineate their effect on the blank shape optimization procedure. From the numerical study it is evident that the optimal blank shape for a part is significantly influenced by the initial anisotropy. The numerical method responds to the material anisotropy and is capable of producing an optimal initial blank shape within few iterations.
\end{abstract}

(c) 2008 Elsevier B.V. All rights reserved.

\section{Introduction}

Blank shape is an important process parameter in sheet metal forming that has a direct impact on the quality as well as the final cost of the formed part. The optimum blank shape uses optimal blank holder force leading to increased formability by reducing tearing and wrinkling and allows achieving near net-shape components. The initial anisotropy in the blank governs the material flow characteristics during deep drawing operation and consequently acts on the quality of the formed part. It dictates the shape of the yield surface and strongly affects the strain distributions obtained during sheet metal forming [1-3]. Anisotropy occurs in blanks primarily due to thermo-mechanical pre-processing and plastic deformation during forming. Plastic deformation induced anisotropy is small compared to initial anisotropy which is the result of a large deformation during initial processing operations such a rolling, extrusion, etc. Due to the complexity involved in its implementation in finite element simulations and increased computation time, generally deformation induced anisotropy is neglected in industrial applications. Moreover, considering only the initial anisotropy produces results in close agreement to the experimental data $[4,5]$.

For many years, the degree of influence of process parameters on the deformation history and their optimum condition has been mainly determined by intuition and experience. Of these process parameters, the initial blank shape for a part is determined mostly by experimental trial and error method. As a result of rapid progress in computing facilities, numerical tools based on the finite element

\footnotetext{
* Corresponding author. Tel.: +351 239790700; fax: +351239790701.

E-mail address: padmanabhan@dem.uc.pt (R. Padmanabhan).
}

method have become common for virtual try-out of sheet metal forming components. Many design approaches have been proposed to determine the initial blank shape, such as the slip-line method [6,7], the characteristics of plane stress, the geometric mapping, inverse approach [8-10], rollback method [11], etc. In all these approaches, either the finite element mesh size was altered during the optimization procedure or only the part's flange area was considered. In this paper, an iterative blank shape optimization procedure, that determines the optimal blank shape for a part, is described. The effect of initial anisotropy in the finite element blank and the orientation of the blanks rolling direction during deep-drawing process are investigated in this study.

\section{Blank shape optimization}

Numerical solutions can provide an insight on the deformation behaviour of blanks subjected to deep drawing. Numerical models are simple to create and modify depending on the requirements, thus eliminating the material wastage. One such solution to determine an optimal blank shape for a sheet metal part is described in this paper. Three numerical tools, DD3IMP, DD3TRIM and NURBS, are used in the optimization procedure to determine optimal blank shape. In Sections $2.1,2.2$, and 2.3 , these numerical tools are briefly described highlighting their contribution to the blank shape optimization procedure.

\subsection{DD3IMP}

Drawing simulations were carried out using the in-house finite element code DD3IMP (contraction of Deep Drawing 3d implicit code). 
DD3IMP is specifically developed to simulate sheet metal forming processes [12-14].

\subsection{DD3TRIM}

In deep-drawing processes, the average element size influences results like draw-in prediction, depending upon the complexity of the final shape of the part. Generally, to accommodate the continuous variation of the blank boundary in optimization procedure, a timeconsuming remeshing procedure is employed. In blank shape optimization, it is important to fix this numerical parameter and avoid meshing procedures. In the strategy presented in this work, a regular and uniform mesh with dimensions large enough to accommodate the probable blank shapes is defined as a base mesh. This base mesh is trimmed with an initial NURBS surface to produce the initial blank. In the optimization iteration, the NURBS surface is updated based on the correction algorithm and the base mesh is trimmed to produce intermediate blank shapes. The base mesh is trimmed using DD3TRIM, a numerical tool developed to trim 3D solid finite element meshes using, among other simple analytical geometrical definitions, NURBS surface representation [15]. DD3TRIM is used in the blank shape optimization procedure to eliminate remeshing.

\subsection{NURBS surface}

A NURBS surface is a geometric modeller extensively used in the design and manufacture of components in aircraft, automobile and shipping industries. NURBS surface is the rational generalization of the tensor-product non-rational B-spline surface and is defined as [16]

$\mathbf{S}(u, v)=\frac{\sum_{i=0}^{n} \sum_{j=0}^{m} w_{i, j} \mathbf{P}_{i, j} N_{i, p}(u) N_{j, q}(v)}{\sum_{i=0}^{n} \sum_{j=0}^{m} w_{i, j} N_{i, p}(u) N_{j, q}(v)}$

where $w_{i, j}$ are the weights, $\mathbf{P}_{i, j}$ form a control net, and $N_{i, p}(u)$ and $N_{j, q}(v)$ are the normalized B-splines of degree $p$ and $q$ in the $u$ and $v$ directions, respectively. The $i$ th $\mathrm{B}$-spline basis function of $p$-degree (order $p+1$ ), denoted by $N_{i, p}(u)$, is defined as

$N_{i, 0}(u)= \begin{cases}1 & \text { if } u_{i} \leqslant u<u_{i+1} \\ 0 & \text { otherwise }\end{cases}$

$N_{i, p}(u)=\frac{u-u_{i}}{u_{i+p}-u_{i}} N_{i, p-1}(u)+\frac{u_{i+p+1}-u}{u_{i+p+1}-u_{i+1}} N_{i+1, p-1}(u)$

defined on the knot vectors

$\mathbf{U}=\left[0,0, \ldots, 0, u_{p+1}, \ldots, u_{n}, 1,1, \ldots, 1\right]$

$\mathbf{V}=\left[0,0, \ldots, 0, v_{q+1}, \ldots, v_{m}, 1,1, \ldots, 1\right]$

where the end knots are repeated with multiplicities $p+1$ and $q+1$, respectively, in order to guarantee that the surface is at least $C^{1}$ continuous at the end knots. The shape of the NURBS surface can be locally modified either by moving control points, by changing knot vectors or by changing the weights. However, in the blank shape optimization procedure, new NURBS surface must be defined during iterations because all points may change their positions.

\subsection{Blank shape optimization procedure}

The NURBS surface used results from a NURBS curve extrusion. The NURBS curve is constructed by interpolating a selected set of points that correspond to nodes on the FE mesh closest to control points of the initial NURBS curve. The interpolated NURBS curve passes through these points. A global interpolation algorithm is used to solve the system of equations retaining the knots and weights, as described in the following paragraph. In this global interpolation procedure, the degree of the NURBS curve is set to 3 because it produced the best results in this study.

Suppose an initial NURBS curve is constructed using an initial set of points $\left\{Q_{k}\right\}, k=0, \ldots, n$. Based on the deformation behaviour of the blank during deep drawing and consequently the blank shape optimization procedure, new positions for these points are determined using a push/pull technique as illustrated in Fig. 1. The initial $\mathbf{X}^{\text {init }}$ and the final $\mathbf{X}^{\text {final }}$ coordinates of the nodes located in selected positions over the blank contour are used to determine intersection points $\mathbf{X}^{\text {inter }}$ on the target contour. The set of points $\left\{Q_{k}\right\}, k=0, \ldots, n$ that the new NURBS surface must interpolate are determined using the push/pull technique defined by

$\mathbf{Q}_{k}=\mathbf{X}_{k}^{\text {init }}+\xi\left(\mathbf{X}_{k}^{\text {inter }}-\mathbf{X}_{k}^{\text {final }}\right) \quad$ with $k=1,2,3$ and $\xi \in[0,1]$

$\xi$ is a damping coefficient used to control the optimization procedure. The new set of points is interpolated with a pth-degree non-rational B-spline curve. Assigning a parameter value $\bar{u}_{k}$ to each $Q_{k}$ will result in a system of $n+1$ linear equations,

$Q_{k}=C\left(\bar{u}_{k}\right)=\sum_{i=0}^{n} N_{i, p}\left(\bar{u}_{k}\right) P_{i}$

for each direction of the Cartesian coordinate system. The chord length method is used for choosing $\bar{u}_{k}$.

Let $d$ be the total chord length,

$d=\sum_{k=1}^{n}\left|Q_{k}-Q_{k-1}\right|$

Then $\bar{u}_{0}=0, \bar{u}_{n}=1$, and the others are calculated using

$\bar{u}_{k}=\bar{u}_{k-1}+\frac{\left|Q_{k}-Q_{k-1}\right|}{d}, \quad k=1, \ldots, n-1$

Solving the system of linear equations will result in a new set of control points defining a new NURBS curve. This curve conforms to a new blank shape and it is extruded to a NURBS surface to demarcate trimming domains for a solid finite element mesh. This procedure is carried out by an in-house code named DD3SHAPE that uses the results from DD3IMP and the current NURBS surface in IGES (Initial Graphics Exchange Specification) format as inputs to produce the new surface, also in IGES format.

The blank shape optimization procedure is illustrated in Fig. 2. The initial process parameters like, the tools geometry, the mechanical properties of the blank, the friction conditions, and the blank holder force are fixed during the optimization procedure. An initial blank shape is selected or determined based on empirical formulae [17], and a corresponding NURBS surface is produced. A regular and uniform mesh with dimensions large enough to accommodate the probable blank shapes is defined as a base mesh. Generally, to accommodate the continuous variation of the nodal coordinates in optimization procedure, a time-consuming remeshing procedure is employed. In the proposed method, the base mesh is always used to cut the initial and intermediate blank shapes in accordance with the initial NURBS surface or those created by the algorithm. This eliminates the influence of the finite element size in the deformation of the blank. The mesh cut using NURBS surface is subjected to deep-drawing simulation. The flange contour of the formed part is compared with the required target contour. If the flange contour is different from target contour, the initial NURBS surface is corrected and a new NURBS surface is produced depending on its deviation. This new NURBS surface is used to trim the base mesh to produce an intermediate blank shape which is subjected to deep-drawing process. The application of the push/pull technique to the NURBS surface allows guaranteeing a smooth surface in each iteration. This 


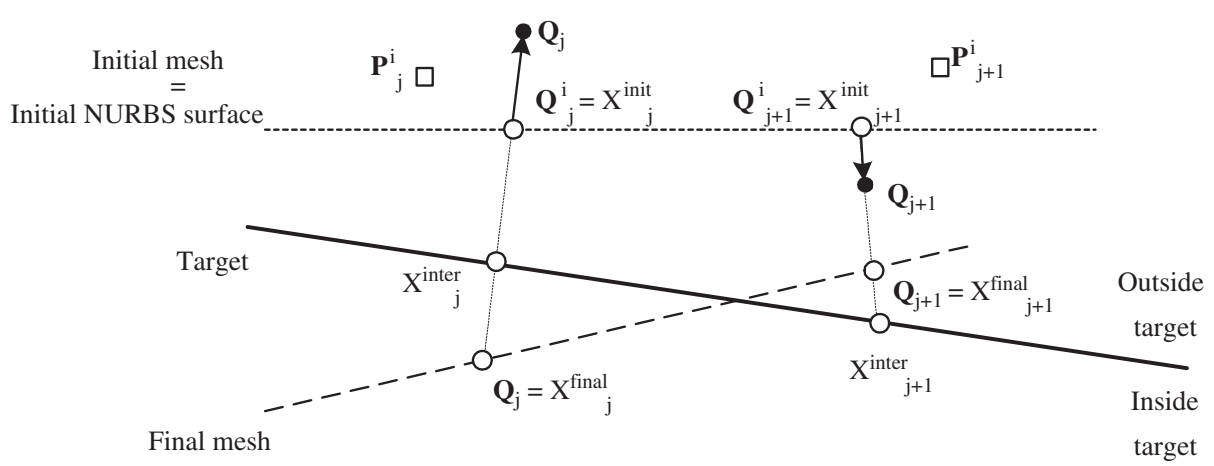

Fig. 1. Example on the application of push/pull technique.

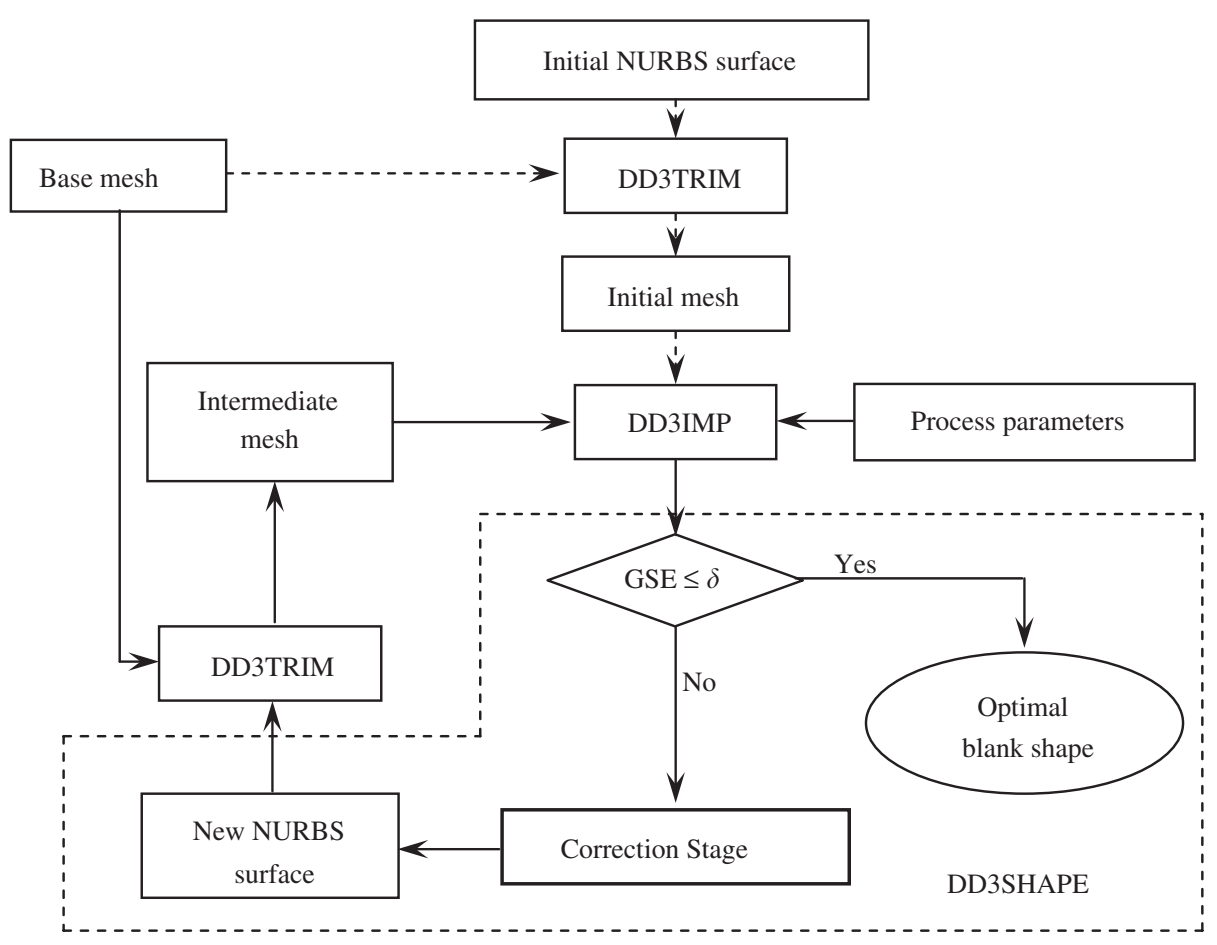

Fig. 2. Blank shape optimization procedure.

procedure is repeated until the deviation between flange contour and target contour falls below a user defined value, $\delta$. The optimization procedure can be fully automated due to the combination of DD3IMP, DD3TRIM and DD3SHAPE (the push/pull technique applied to the NURBS surface).

\subsection{Shape error}

In order to quantify the deviation between the flange and the target contours, a geometrical measure namely, geometrical shape error, is used. Geometrical shape error (GSE), expressed in $\mathrm{mm}$, is defined as the root mean square of the shape difference between the target shape and the deformed shape as in the following equation [8]:

$\mathrm{GSE}=\sqrt{\frac{1}{n} \sum_{i=1}^{n}\left|\mathbf{X}^{\text {inter }}-\mathbf{X}^{\text {final }}\right|^{2}}$

where $\mathbf{X}^{\text {inter }}$ and $\mathbf{X}^{\text {final }}$ are the coordinates of the predefined set of points at the intersection with the target contour and at the end of the forming process, respectively. $n$ is the number of control points used in the initial NURBS surface and is retained during the optimization procedure. When the GSE reaches a value less than a predetermined value for a required accuracy in the flange shape, $\delta$, the iterative procedure is stopped because the optimal blank shape for the part has been obtained. The GSE defines the stopping criterion for the blank shape optimization procedure. For the examples presented in the following sections, inaccuracy in flange shape $(\delta)$ less than $0.5 \mathrm{~mm}$ is used as stopping criterion.

Large initial blank shape results in large GSE in the first iteration and hence the correction algorithm underestimates the first intermediate blank shape. In the second iteration, due to much smaller flange contour compared to the required target contour, the correction algorithm overestimates the second intermediate blank shape. This strong variation in the intermediate blank shapes increases the number iteration to achieve the required accuracy. Hence, the damping coefficient presented in Eq. (4) was used to control the strong variation in intermediate blank shapes. For the tool geometries studied, a damping coefficient of 0.6 leads to fast convergence of the blank shape optimization algorithm. 
a
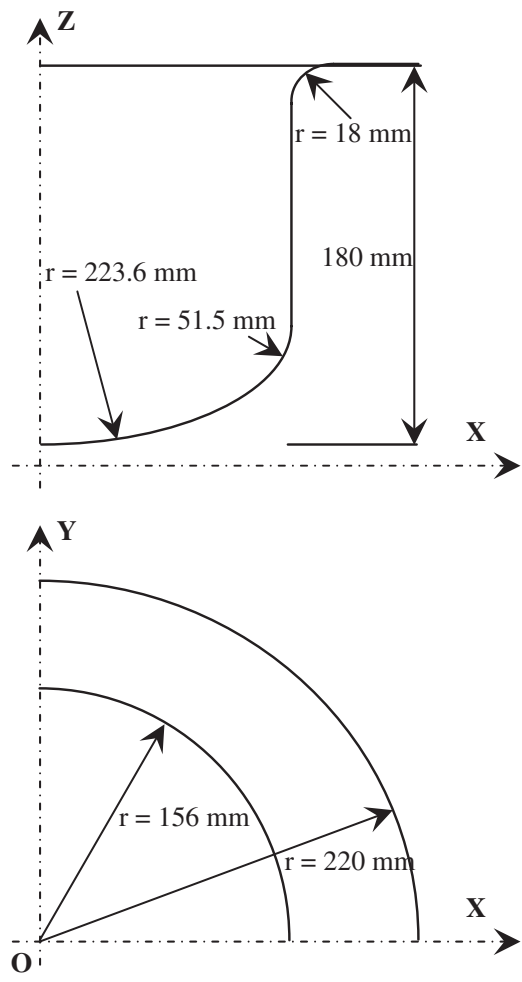

b

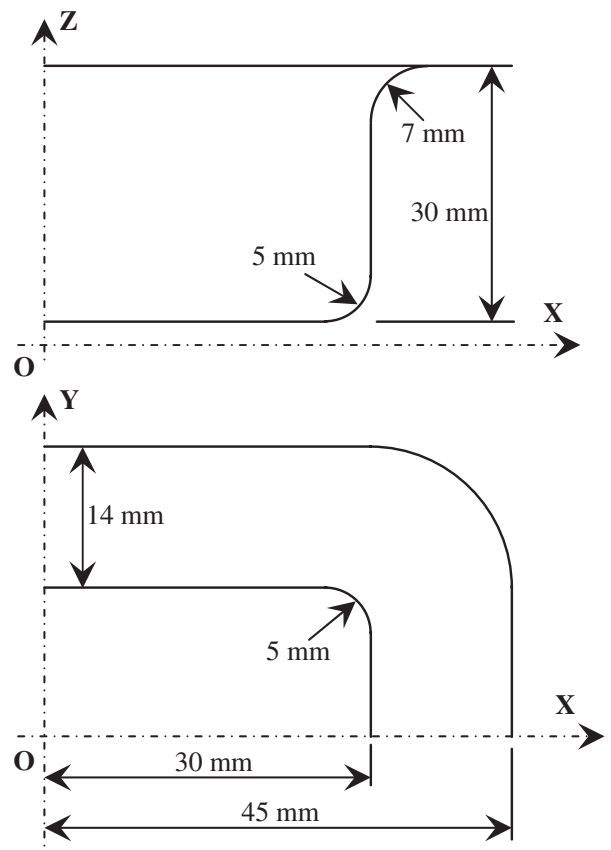

Sketch drawn not to scale

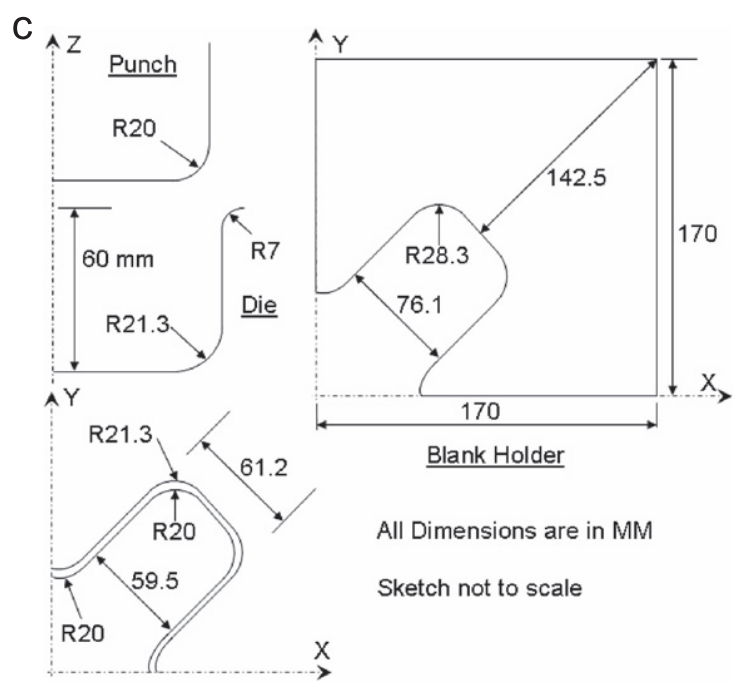

Fig. 3. (a) Circular cup, (b)rectangular cup, (c) cross tool geometries.

\section{Example geometries}

\subsection{Geometry of the cups}

Most parts subjected to stamping have complex geometries and results in differential material flow characteristics around the part. To capture the influence of geometry, two shapes were extensively utilized in this study, a circular cup and a rectangular cup. Cross tool geometry [18], used by Renault to benchmark new materials, is used to analyse the influence of contact friction condition on the optimal blank shape. The geometry of the circular cup, the rectangular cup and the cross tool is presented in Fig. 3(a), (b) and (c), respectively. The blank thickness used for circular cup is 3.5 and $0.8 \mathrm{~mm}$ for both rectangular cup and cross tool.

\subsection{Material anisotropic properties}

The blank shape optimization procedure described in Section 2.4 capture the material flow characteristics into account since it is based on the FE simulation results. The material flow characteristic is in turn governed by the initial anisotropy in addition to other material properties. A good description of the anisotropy in the rolled sheets is essential for a quantitative approach to deep-drawing process simulations. Phenomenological plasticity theories consider initial anisotropy through a yield criterion. A number of such yield criterions have so far been proposed by Hill [19], Barlat [20,21], Karafillis and Boyce [22], Drucker [23], etc. and equally studies have been carried out to compare these yield functions, for instance [24-29]. Hill' 48 yield function is widely used in finite element simulation 
studies and has been the primary choice for explaining some phenomena associated with anisotropic plasticity, especially for steel blanks. Hence in this work, the anisotropy of the rolled blank is described by the analytical function in the quadratic form postulated by Hill:

$$
\begin{aligned}
\bar{\sigma}^{2}= & F\left(\sigma_{22}-\sigma_{33}\right)^{2}+G\left(\sigma_{33}-\sigma_{11}\right)^{2}+H\left(\sigma_{11}-\sigma_{22}\right)^{2} \\
& +2 L \sigma_{23}^{2}+2 M \sigma_{31}^{2}+2 N \sigma_{12}^{2}
\end{aligned}
$$

where the numerals 1, 2, 3 stand for the orthotropic directions. $F, G$, $H, L, M, N$ are the six material parameters that depend on the flow stresses under uniaxial and shear loading in the principal directions, respectively, and implicitly assumes that hydrostatic stress states lead to no yielding and no plastic deformation.

An anisotropic yield criterion proposed by Cazacu and Barlat [30] (CB2001) was also used to outline the influence of material model in the earing prediction. To describe yielding of orthotropic materials, a generalization of the effective stress tensor was developed. These generalized stress invariants are then used to extend the Drucker's isotropic yield criterion to orthotropy,

$\bar{\sigma}=\left\{27\left[\left(J_{2}^{0}\right)^{3}-c\left(J_{3}^{0}\right)^{2}\right]\right\}^{1 / 6}$

where $J_{2}^{0}$ and $J_{3}^{0}$ are the second and third generalized invariants of the effective deviatoric stress tensor $\boldsymbol{\Sigma}=\boldsymbol{\sigma}^{\prime}-\mathbf{X}$, where $\boldsymbol{\sigma}^{\prime}$ is the deviatoric stress tensor and $\mathbf{X}$ is the back stress tensor. The invariants are defined as

$$
\begin{aligned}
J_{2}^{0}= & \frac{a_{1}}{6}\left(\Sigma_{x x}-\Sigma_{y y}\right)^{2}+\frac{a_{2}}{6}\left(\Sigma_{y y}-\Sigma_{z z}\right)^{2}+\frac{a_{3}}{6}\left(\Sigma_{x x}-\Sigma_{z z}\right)^{2} \\
& +a_{4} \Sigma_{x y}^{2}+a_{5} \Sigma_{x z}^{2}+a_{6} \Sigma_{y z}^{2}
\end{aligned}
$$

and

$$
\begin{aligned}
J_{3}^{0}= & \frac{1}{27}\left(b_{1}+b_{2}\right) \Sigma_{x x}^{3}+\frac{1}{27}\left(b_{3}+b_{4}\right) \Sigma_{y y}^{3}+\frac{1}{27}\left[2\left(b_{1}+b_{4}\right)-b_{2}-b_{3}\right] \Sigma_{z z}^{3} \\
& -\frac{1}{9}\left(b_{1} \Sigma_{y y}+b_{2} \Sigma_{z z}\right) \Sigma_{x x}^{2}-\frac{1}{9}\left(b_{3} \Sigma_{z z}+b_{4} \Sigma_{x x}\right) \Sigma_{y y}^{2} \\
& -\frac{1}{9}\left[\left(b_{1}-b_{2}+b_{4}\right) \Sigma_{x x}+\left(b_{1}-b_{3}+b_{4}\right) \Sigma_{y y}\right] \Sigma_{z z}^{2} \\
& +\frac{2}{9}\left(b_{1}+b_{4}\right) \Sigma_{x x} \Sigma_{y y} \Sigma_{z z}-\frac{\Sigma_{x z}^{2}}{3}\left[2 b_{9} \Sigma_{y y}-b_{8} \Sigma_{z z}-\left(2 b_{9}-b_{8}\right) \Sigma_{x x}\right] \\
& -\frac{\Sigma_{x y}^{2}}{3}\left[2 b_{10} \Sigma_{z z}-b_{5} \Sigma_{y y}-\left(2 b_{10}-b_{5}\right) \Sigma_{x x}\right] \\
& -\frac{\Sigma_{y z}^{2}}{3}\left[\left(b_{6}+b_{7}\right) \Sigma_{x x}-b_{6} \Sigma_{y y}-b_{7} \Sigma_{z z}\right]+2 b_{11} \Sigma_{x y} \Sigma_{y z} \Sigma_{x z}
\end{aligned}
$$

For 3D stress conditions this criterion involves 18 anisotropy parameters $\left(a_{k}, k=1,6 ; b_{k}, k=1,11\right.$; and $\left.-3.375 \leqslant c \leqslant 2.25\right)$.

The identification of the Cazacu and Barlat (CB2001) anisotropy parameters is complex and a special in-house code (DD3MAT) developed for this purpose was used to guarantee the best fit to the experimental data [31]. The identification of the constitutive parameters involved: (i) uniaxial tensile tests up to localized necking with samples taken at intervals of $15^{\circ}$ orientation with respect to the rolling direction and (ii) monotonic simple shear tests up to $50 \%$ shear with samples taken at $0^{\circ}, 45^{\circ}$ and $90^{\circ}$ orientations with respect to the rolling direction. This means that 15 experimental results were used to identify the parameters (seven $r$-values, seven uniaxial yield stresses and three simple shear stresses). The minimization strategy implemented in the special purpose in-house code DD3MAT

\begin{tabular}{|c|c|c|c|c|c|c|c|c|c|}
\hline \multicolumn{10}{|l|}{ (a) } \\
\hline & \multicolumn{2}{|l|}{$\mathrm{F}$} & G & \multicolumn{3}{|l|}{$\mathrm{H}$} & $\mathrm{L}$ & M & $\mathrm{N}$ \\
\hline DC06 & \multirow{2}{*}{\multicolumn{2}{|c|}{$\begin{array}{l}0.2635 \\
0.51274\end{array}$}} & 0.2833 & \multirow{2}{*}{\multicolumn{3}{|c|}{$\begin{array}{l}0.7169 \\
0.5025\end{array}$}} & 1.5 & 1.5 & 1.2795 \\
\hline DP600 & & & 0.4975 & & & & 1.5 & 1.5 & 1.2729 \\
\hline \multirow{2}{*}{$\begin{array}{l}\text { (b) } \\
\text { DC06 }\end{array}$} & & & & & & & & & \\
\hline & $\begin{array}{l}a_{1} \\
1.2076 \\
b_{4} \\
0.2672\end{array}$ & $\begin{array}{l}a_{2} \\
0.8765 \\
b_{5} \\
2.0024\end{array}$ & $\begin{array}{l}a_{3} \\
0.9754 \\
b_{6} \\
1.0\end{array}$ & $\begin{array}{l}a_{4} \\
1.0767 \\
b_{7} \\
1.0\end{array}$ & $\begin{array}{l}a_{5} \\
1.0 \\
b_{8} \\
1.0\end{array}$ & $\begin{array}{l}a_{6} \\
1.0 \\
b_{9} \\
1.0\end{array}$ & $\begin{array}{l}b_{1} \\
0.6101 \\
b_{10} \\
1.1146\end{array}$ & $\begin{array}{l}b_{2} \\
4.1535 \\
b_{11} \\
1.0\end{array}$ & $\begin{array}{l}b_{3} \\
3.7750 \\
c \\
0.4276\end{array}$ \\
\hline DP600 & $\begin{array}{l}a_{1} \\
0.9861 \\
b_{4} \\
1.2194\end{array}$ & $\begin{array}{l}a_{2} \\
1.0083 \\
b_{5} \\
0.5119\end{array}$ & $\begin{array}{l}a_{3} \\
1.0215 \\
b_{6} \\
1.0\end{array}$ & $\begin{array}{l}a_{4} \\
0.8890 \\
b_{7} \\
1.0\end{array}$ & $\begin{array}{l}a_{5} \\
1.0 \\
b_{8} \\
1.0\end{array}$ & $\begin{array}{l}a_{6} \\
1.0 \\
b_{9} \\
1.0\end{array}$ & $\begin{array}{l}b_{1} \\
0.9253 \\
b_{10} \\
0.5951\end{array}$ & $\begin{array}{l}b_{2} \\
1.1438 \\
b_{11} \\
1.0\end{array}$ & $\begin{array}{l}b_{3} \\
1.2099 \\
c \\
1.1547\end{array}$ \\
\hline
\end{tabular}
can be used with any number of experimental values, for both yield criteria. However, the Hill'48 parameters were determined following the classical methodology using only the experimental $r$-values $r_{0}, r_{45}$ and $r_{90}$ which are the ratios of the width-to-thickness strains
Table 1

Material properties of mild steel (DC06) and dual-phase steel (DP600)

\begin{tabular}{lll}
\hline Properties & DC06 & DP600 \\
\hline$E$ & $210.0 \mathrm{GPa}$ & $210.0 \mathrm{GPa}$ \\
$v$ & 0.3 & 0.3 \\
$Y_{0}$ & $123.6 \mathrm{MPa}$ & $330.3 \mathrm{MPa}$ \\
$K$ & $529.5 \mathrm{MPa}$ & $1093 \mathrm{MPa}$ \\
$n$ & 0.268 & 0.187 \\
\hline
\end{tabular}

Table 2

Material parameters for (a) Hill'48 models and (b) CB2001 models [32,33].

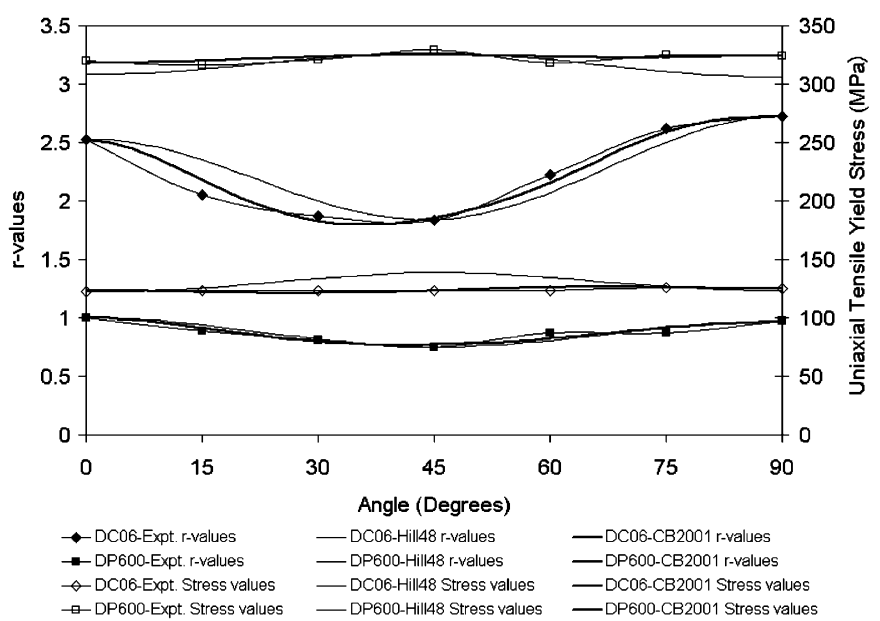

Fig. 4. Anisotropy of the $r$-values and the uniaxial tensile stresses for the DC06 and DP600 steel. Comparison between experimental results and Hill'48 and Cazacu and Barlat'01 (CB2001) yield criteria.

during a tensile test, at $0^{\circ}, 45^{\circ}$, and $90^{\circ}$, respectively, to the rolling direction. DC06 steel exhibits a weak planar anisotropy of the flow stress with $r_{0}=2.53, r_{45}=1.84$ and $r_{90}=2.72$, and DP600 is nearly isotropic regarding the flow stress with $r_{0}=1.01, r_{45}=0.76$ and $r_{90}=0.98$. The material properties of DC06 and DP600 blanks used in the simulation are listed in Table 1.

In Table $1, E$ is the Young's modulus, $v$ is the Poisson's ratio, $Y_{0}$ is the yield stress, $n$ and $K$ are the material properties according to the Swift law which describes isotropic work-hardening equation:

$\sigma=K\left(\varepsilon_{0}+\bar{\varepsilon}^{\mathrm{P}}\right)^{\mathrm{n}}$

where $\sigma$ is the flow stress, $\bar{\varepsilon}^{\mathrm{P}}$ is the equivalent plastic strain and $\varepsilon_{0}=\left(Y_{0} / K\right)^{1 / n}$. The material anisotropy parameters used in different material models are listed in Table 2 [32,33]. Fig. 4 highlights the difference in modelling the anisotropic behaviour of the steels using two yield criterions. As shown, the Cazacu and Barlat (CB2001) yield 
criterion closely describes the experimental anisotropic behaviour, both interms of $r$-values and uniaxial tensile stresses.

Initial process parameters were chosen based on empirical relations and optimal values. A blank holder force of 800 and 50,000 N was used for DC06 rectangular cup and circular cup, respectively, and a friction coefficient of 0.08 was used in both the cases. For DP600, the blank holder forces used were 2000 and $70,000 \mathrm{~N}$ for rectangular cup and circular cup, respectively. The friction coefficients of 0.02 and 0.08 were used for rectangular cup and circular cup, respectively. The values of the process parameters remained the same for all simulations within a blank shape optimization cycle.

\section{Discussion on results}

\subsection{Influence of material properties description}

Anisotropy has strong influence on the material flow and hence formability of the blank. The initial anisotropy prevalent in the preprocessed sheet segment greatly influences subsequent deformation such as deep drawing. The anisotropy results in differential strength in different directions of the blank. The differential strength effect induces significant difference in material flow and hence results in earing profile in the formed part. Fig. 5 shows the initial blank contour

a

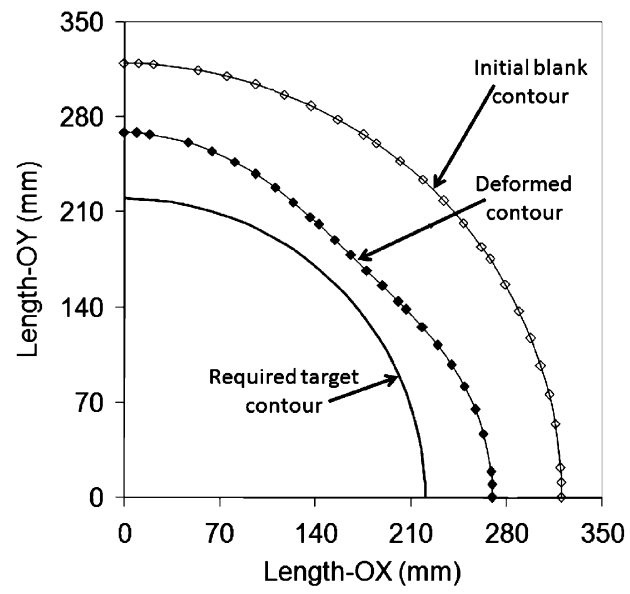

b

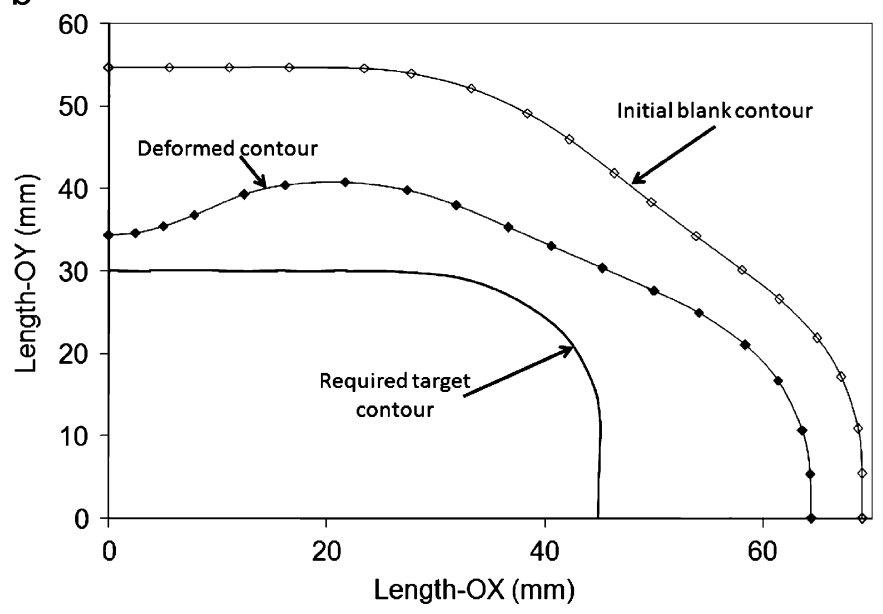

Fig. 5. (a) Initial and final blank contours of circular cup. (b) Initial and final blank contours of rectangular cup.

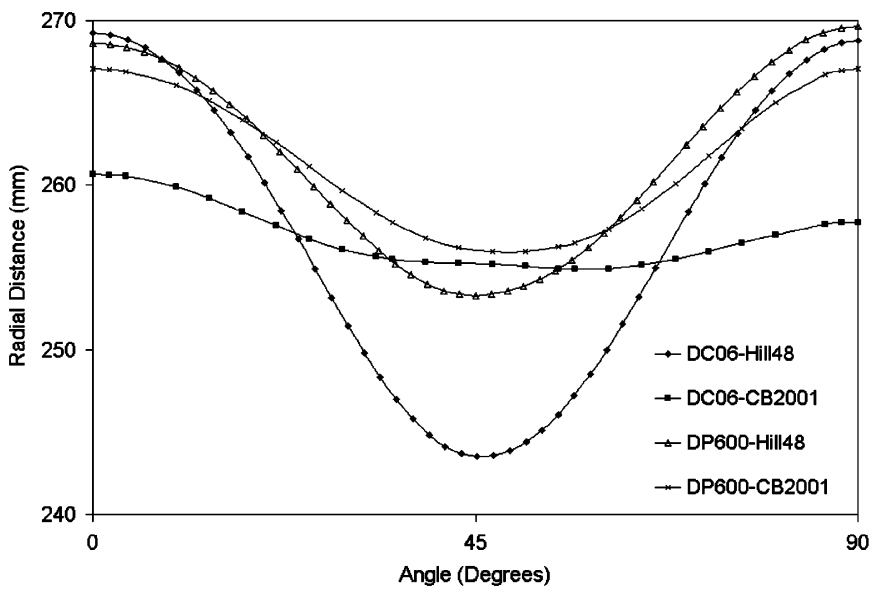

Fig. 6. Draw-in of the blanks after circular cup deep drawing simulations.

and the contour after deep drawing DC06 steel using Hill'48 yield criterion. To demonstrate the robustness of described optimization algorithm, large initial blanks were considered for both geometries assuming ideal flow behaviour without thinning. Fig. 5(a) shows the initial blank contour, with a radius of $320 \mathrm{~mm}$, and final contour after deep drawing circular cup. An optimal blank shape required to achieve a given target contour depends on the material properties and the geometry of the part. Fig. 5(b) shows the initial blank contour determined using empirical formulae [17] and the final contour after forming the rectangular cup. The blank shape required to achieve the target contour will be different from the blank shape predicted empirically.

In both geometries, using initial blank contour, excess flange was observed due to ideal forming assumption. In actual practice, flow characteristics differ at various locations in a part according to the intrinsic material properties (see Fig. 4) leading to variation in thickness and consequently earing. Earing is predominantly affected by the anisotropic coefficients ( $r$-value), yield stress, drawing ratio and strain-hardening exponent. A number of models have been specifically developed to describe anisotropic phenomena in different materials [2,3]. For example, in a circular cup, four to six ears will be formed in the part depending on the material and the numerical model [34]. Earing is undesirable in a formed part as the part needs trimming to produce the required flange and consequently excessive material loss.

Fig. 6 shows the radius corresponding to the earing profile of the circular cup, using DC06 and DP600 material models, at the end of first iteration in blank shape optimization procedure. The influence of anisotropy in the blank significantly affects the flow characteristics, and hence the flange contour, in deep-drawing process. Though the difference in the material flow prediction between Hill' 48 [19] and CB2001 [30] is evident, the trend appears to be similar for both materials. More material flow occurs along $45^{\circ}$ to rolling direction compared to that along rolling direction and $90^{\circ}$ to rolling direction. For the nearly isotropic regarding the flow stress (DP600 steel), the Hill'48 yield criteria lead to similar results to the ones obtained with CB2001 yield criteria. However, for the DC06 steel, due to the anisotropy in flow stress, the material flow predictions with both models are rather different. Large deviations are observed along the rolling and transverse directions due to under-estimation and increased draw-in along $45^{\circ}$ to rolling direction, due to overestimation using Hill'48 model. This led to the formation of ears along rolling direction and transverse direction for the Hill'48 model, while CB2001 predicts a more uniform material flow, more in accordance with experimental results (Fig. 4). 


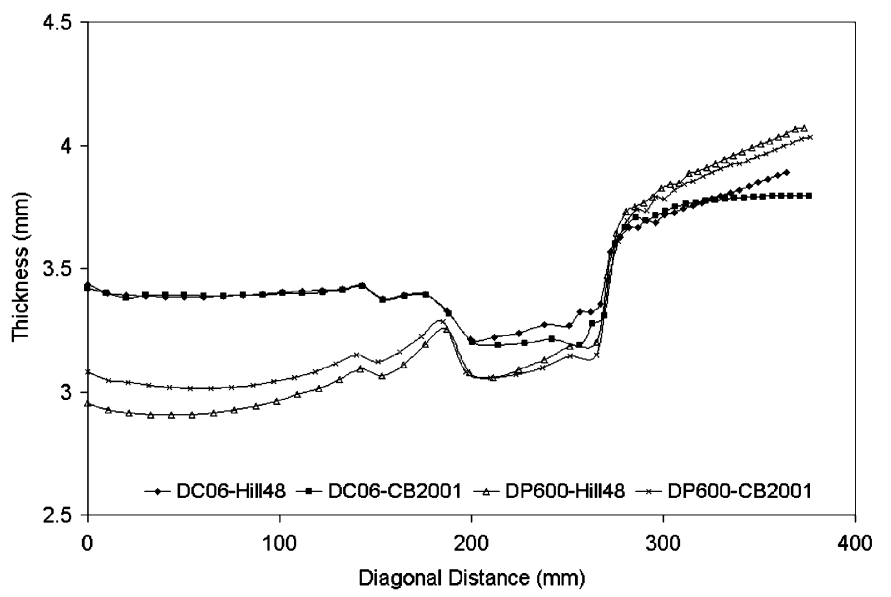

Fig. 7. Thickness variation in circular cups along diagonal direction for (a) DC06 and (b) DP600 steels.

\subsection{Thickness variation}

Distinct difference in the flow characteristics and hence the flange contour is inevitable due to various material parameters used in the models. With the strategy used to identify the anisotropy parameters, the Hill'48 yield criteria takes into account the anisotropy of Lankford values while CB2001 also considers the anisotropy of flow stress. The mechanical behaviour description of a material is important to simulate deep-drawing process accurately. Discrepancy in the result could be attributed to inaccuracies in capturing the material behaviour under different flow characteristics. It can be significant in anisotropic materials such as mild steel (DC06). As a consequence to this variation in material flow, inhomogeneous thickness distribution occurs in the formed part. Most defects occur in sheet metal formed parts due to thinning and hence thickness distribution has direct impact on the part's quality. To guarantee a good solution, the thickness variation in the formed part should be minimized. As explained in the previous paragraph, varied earing profile is formed in the deep drawn part depending on the material properties. This is due to constricted material flow into the die cavity along certain directions and free flow along others. Fig. 7 shows the thickness variation in DC06 and DP600 steel circular cups along diagonal direction for Hill'48 and CB2001 yield criterions at the end of first iteration in blank shape optimization procedure. The draw-in along diagonal direction is more compared to rolling and transverse directions. In DC06 steel cup, using Hill'48 yield criteria results in increased material flow along diagonal direction and hence the thickness distribution is improved. While using CB2001 yield criteria, marginal difference in the material flow along different directions is observed. As a result, pronounced variation in the thickness is observed along diagonal direction, especially at the cup wall and flange area of the formed cup. In DP600 steel cup, Hill'48 yield criteria predict thinning while CB2001 model predicts an improved thickness distribution. The large difference in thickness variation between the two steels can be attributed to the anisotropic nature of DC06 steel and the mechanical behaviour description by two different yield criterions.

For anisotropic materials such as DC06 steel, the orientation of blank rolling direction can influence thickness distribution. Fig. 8(a) illustrates the thickness distribution in the rectangular cups along OX direction for $0^{\circ}, 45^{\circ}$, and $90^{\circ}$ blank orientations for DC06 steel using Hill'48 yield criteria, at the end of first iteration in blank shape optimization procedure. The thickness remained the same across the bottom of the cup and reduced along the punch radius and the cup wall in all the three cases. The thickness increased at the flange sec-

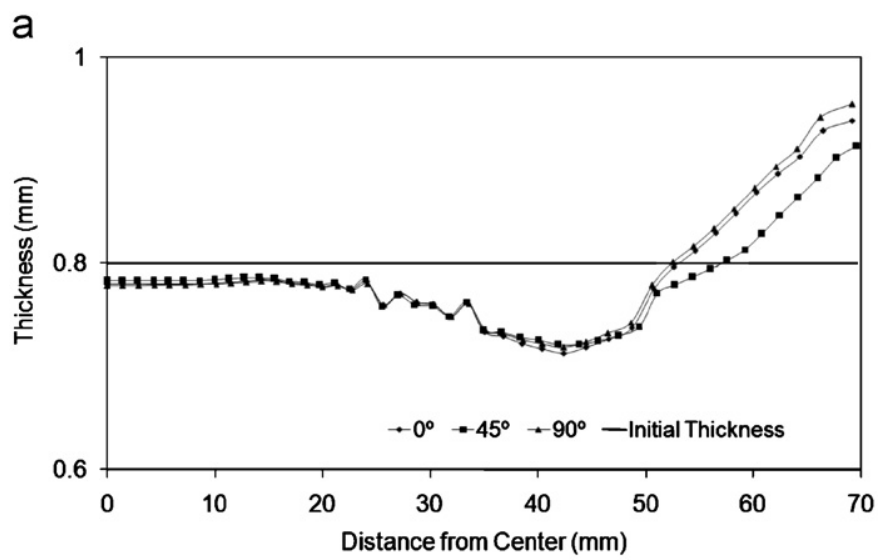

b

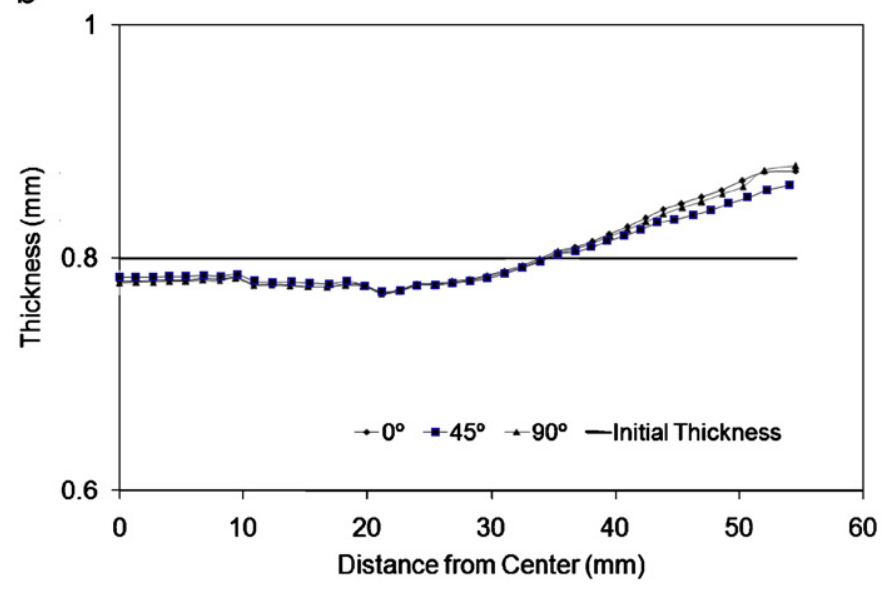

Fig. 8. (a) Thickness variation in rectangular cups along $O X$ direction. (b) Thickness variation in rectangular cups along $O Y$ direction.

tion due to radial compression caused by the draw-in. The thickness increase at the flange is similar for $0^{\circ}$ and $90^{\circ}$ orientations and lower for $45^{\circ}$ orientation of the blank. This is due to increased thickness strain along $45^{\circ}$ to the rolling direction of the blank. When the rolling direction of the blank is oriented at $45^{\circ}$ to $X$-axis material flow more along the $X$ - and $Y$-axis, hence the thickness at the flange and earing is less in this case. This clearly describes the improved thickness strain distribution along $45^{\circ}$ to rolling direction as more material flow occurs in this direction (Fig. 6).

As shown in Fig. 8(b), the variation in thickness along OY direction is marginal for DC06 steel blank. The deviation in thickness is observed only in the flange area while orienting the rolling direction at $45^{\circ}$ to $O X$ axis.

\subsection{Optimal blank shape}

The objective of this study is to determine the optimal blank shape for a rectangular cup and a circular cup and to investigate the influence of rolling direction orientation on the final blank shape for the parts. The influence of the rolling direction on the optimal blank shape depends on the flow characteristics of the material. The blank shape optimization study was performed considering both yield criteria to highlight its sensitivity to initial anisotropy in the blank.

Fig. 9 shows the optimal blank shapes obtained with the described optimization procedure, for different rolling direction orientations in DC06 steel using both yield criteria. Significant difference in blank shapes is evident from the results with Hill' 48 model. Fig. 9(a) shows 
a

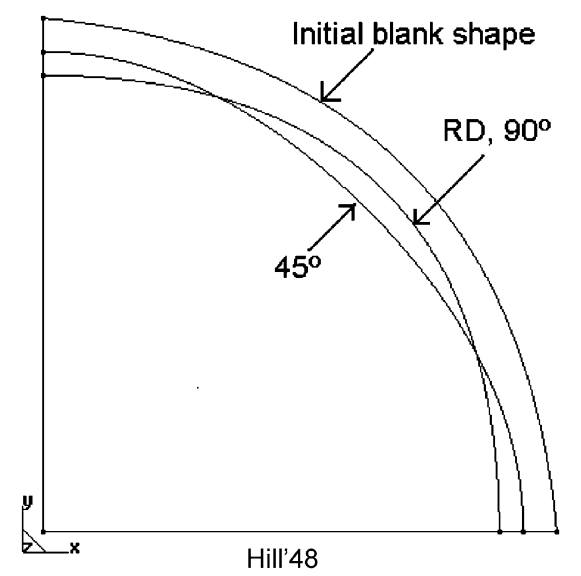

b

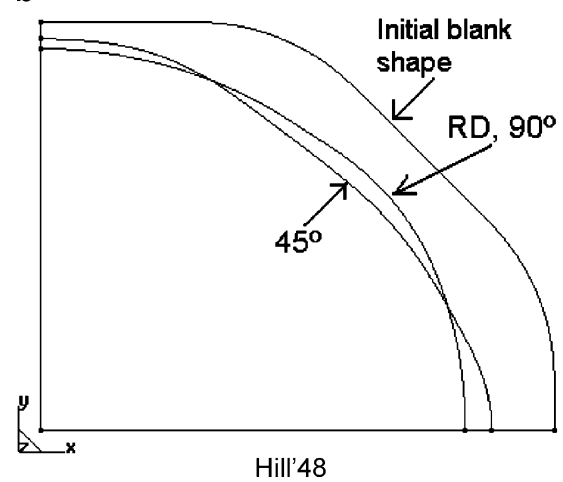

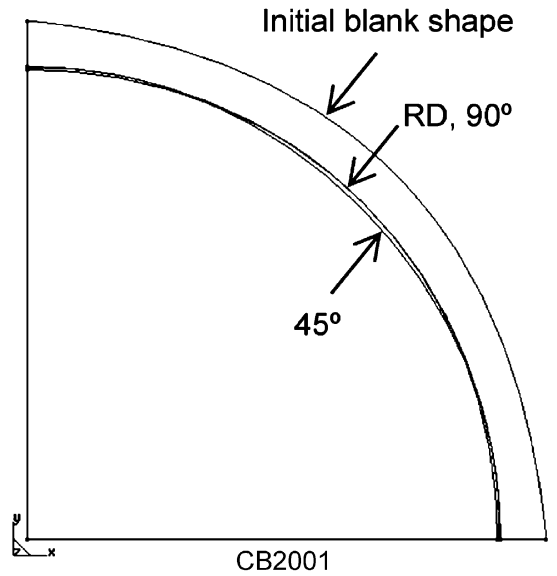

Initial blank

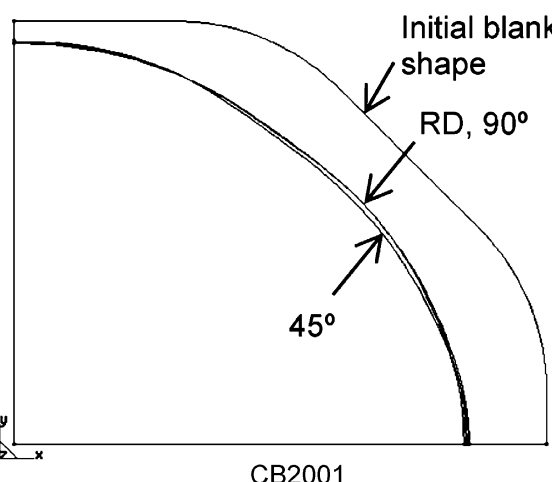

CB2001

Fig. 9. Optimal DCO6 blank shapes for (a) circular cup and (b) rectangular cup.

a

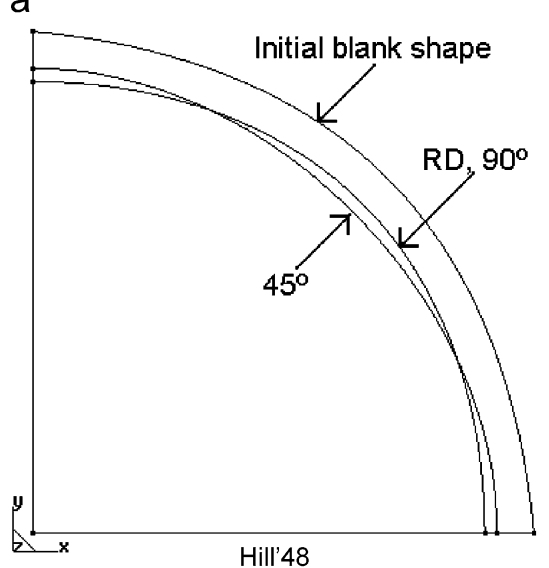

b

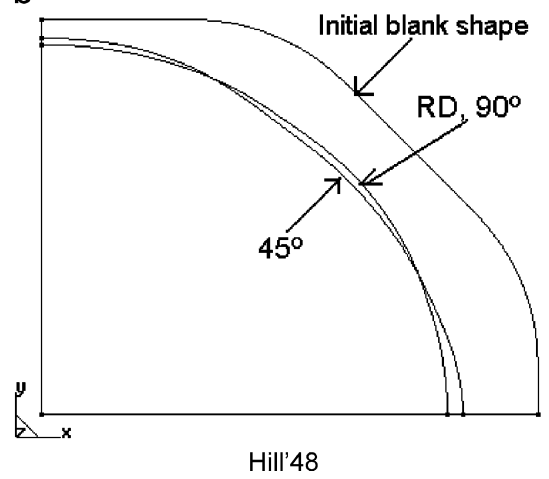

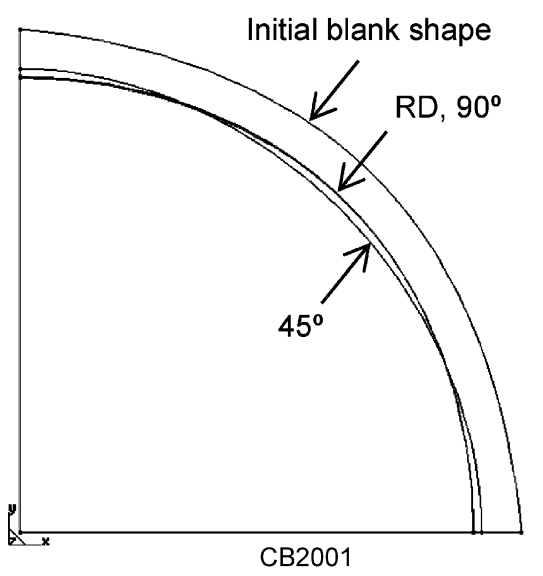

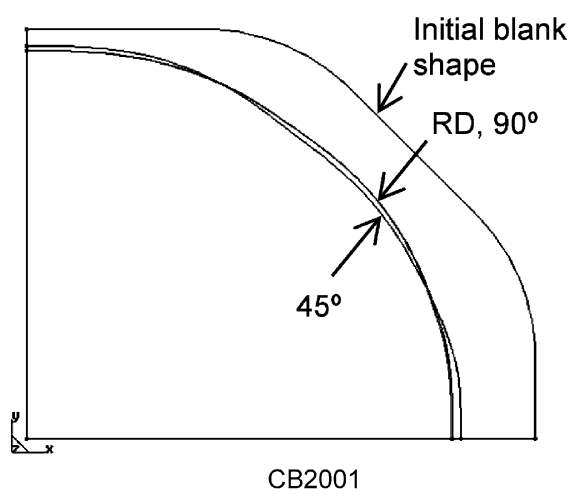

Fig. 10. Optimal DP600 blank shapes for (a) circular cup and (b) rectangular cup. 
the optimal blank shapes for circular cups and Fig. 9(b) for the rectangular cups. When the rolling direction of the blank is parallel $\left(0^{\circ}\right)$ and perpendicular $\left(90^{\circ}\right)$ to $X$-axis $(O X)$, the optimal blank shape resembles nearly the same. When the rolling direction of the blank is oriented $45^{\circ}$ to $X$-axis $(O X)$, an optimal blank larger along the axes and shorter along the diagonal is required for the cup, in particular when Hill'48 yield criterion is used. The results are in good agreement with the fact that according to the Hill'48 yield criterion more flow occurs along $45^{\circ}$ to the rolling direction. Description of DC06 material behaviour using CB2001 leads to more uniform flow characteristics resulting in marginal difference on the optimal blank shapes for both circular and rectangular cups. This is consistent to the result obtained while analysing the draw-in, see Fig. 6 . The over-estimation of flow stress along diagonal direction led to relatively less drawin in this direction. Therefore, negligible difference on the optimal blank shapes for different rolling direction orientations was observed while using CB2001 yield criterion. Similarly, Fig. 10 shows the optimal blank shapes for DP600. Fig. 10(a) shows the blank shapes for circular cups and Fig. 10(b) for rectangular cups. DP600 with an $r$ value close to 1 requires marginally different blank shapes for different rolling direction orientations. For DP600 steel CB2001 yield criterion closely describes the material behaviour both in terms of $r$-values and uniaxial tensile yield stress values. Small difference, as shown in the figures, is observed in the optimal blank shapes when using different rolling direction orientations.

One of the tangible benefits observed while using the blank shape optimization procedure is improvement in thickness distribution in the formed part. Even with the Hill'48 yield criteria that predicts thinning when subjected to biaxial stress state, this improvement in thickness occurs. Fig. 11(a) shows the thickness variation in DC06 steel circular cup using Hill'48 yield criteria. The thickness distribution improved over iterations as shown in the figure using 1 st and 4 th iteration results, along the diagonal direction $\left(45^{\circ}\right.$ to rolling direction). Due to the use of optimal blank, the flow characteristics is enhanced and significantly improved thickness distribution occurs in the formed part as shown in the figure. Similarly, Fig. 11(b) shows the thickness variation in DP600 steel circular cup using Hill'48 yield criteria. The thickness variation reduced in the 4th iteration compared to the 1 st iteration result. The thickness along the cup wall is significantly improved due to the use of optimal blank shape.

\subsection{Efficiency of the blank shape optimization procedure}

In spite of the influence of the initial anisotropy and consequently earing tendency of the blank, the described blank shape optimization method is capable of determining optimal blank shape for a formed part within few iterations. This is amply demonstrated with two geometries: rectangular and circular cup examples. Fig. 12 shows the evolution of GSE for circular and rectangular cups for $0^{\circ}, 45^{\circ}$ and $90^{\circ}$ rolling direction orientations of the blank. Large GSE is observed in the initial stages of blank shape optimization procedure due to large initial blank (Fig. 5) determined using empirical solutions. The shape error reduces significantly over optimization iterations as shown in the figure. In both the cases, the GSE reduced to less than $0.5 \mathrm{~mm}$ within four iterations.

\subsection{Effect of friction between forming tools and blank on the optimal blank shape}

Individual process parameter has influence on the determination of optimal blank shape for a part. The contribution of friction between forming tools and the blank on the optimal blank shape for an industrially relevant geometry is illustrated using Fig. 13. The cross tool geometry is used by Renault car manufacturer to bench mark new materials. This geometry produces most of the industrial
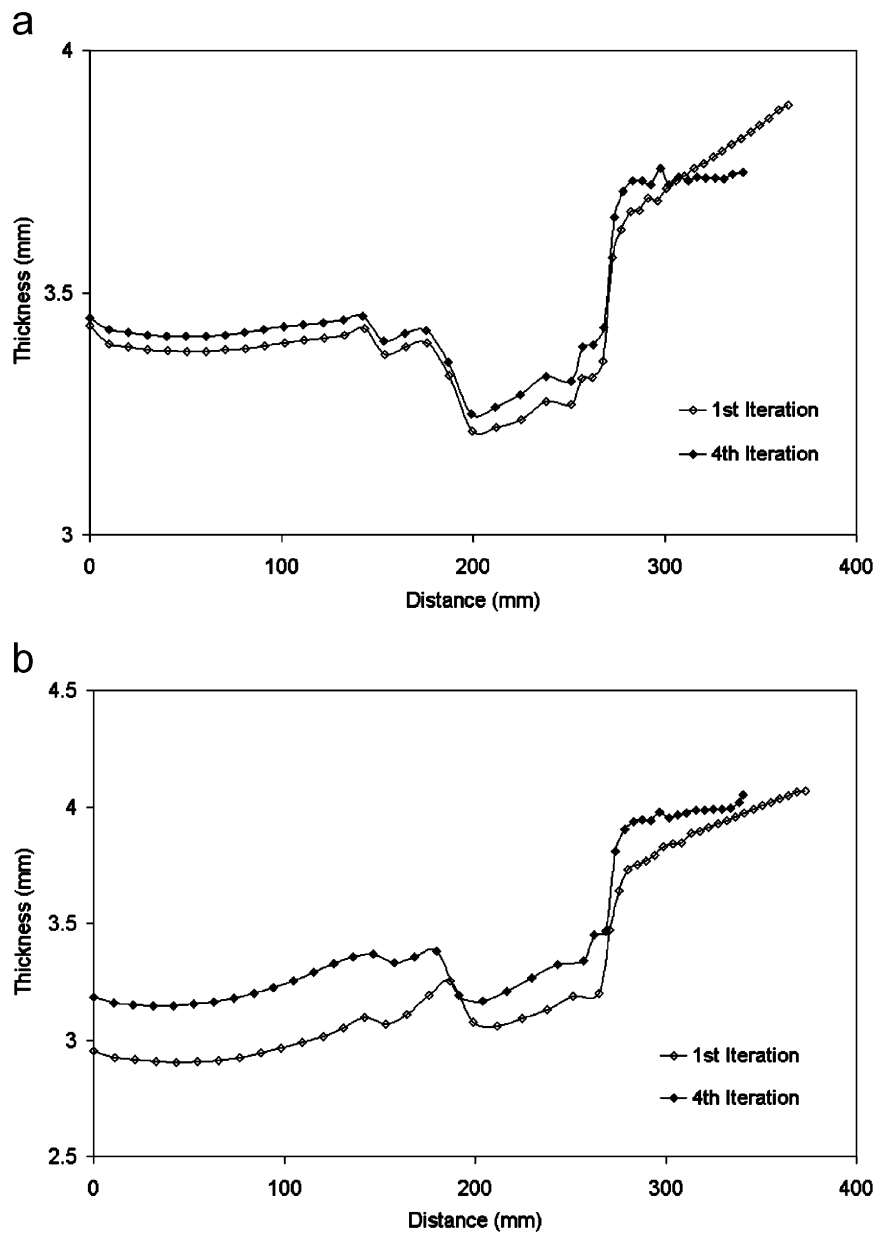

Fig. 11. Improvement in thickness over iterations (a) DC06 and (b) DP600 circular cups.

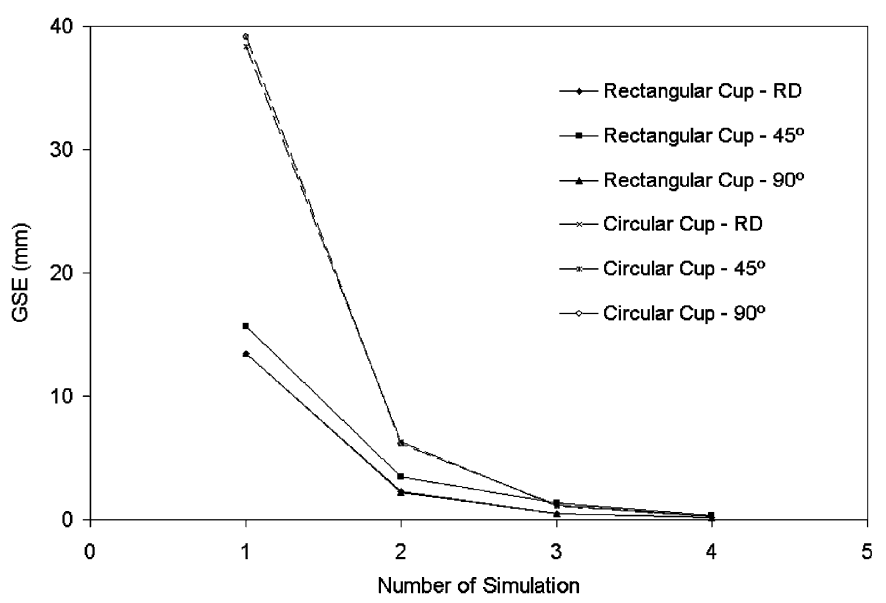

Fig. 12. Evolution of geometric shape error for the two geometries.

strain paths such as simple tension, plane strain, shear and biaxial stretching, etc. The required flange contour for the cross tool is $100 \times 100 \mathrm{~mm}^{2}$ with an arc tangent of $50 \mathrm{~mm}$ radius at the corner, after a draw depth of $60 \mathrm{~mm}$. Simulations were carried out for three contact friction conditions ( $\mu=0.08,0.11$ and 0.14 ). High friction between the forming tools and the blank results in restricted material flow, while a low friction results in smooth flow of material. This 


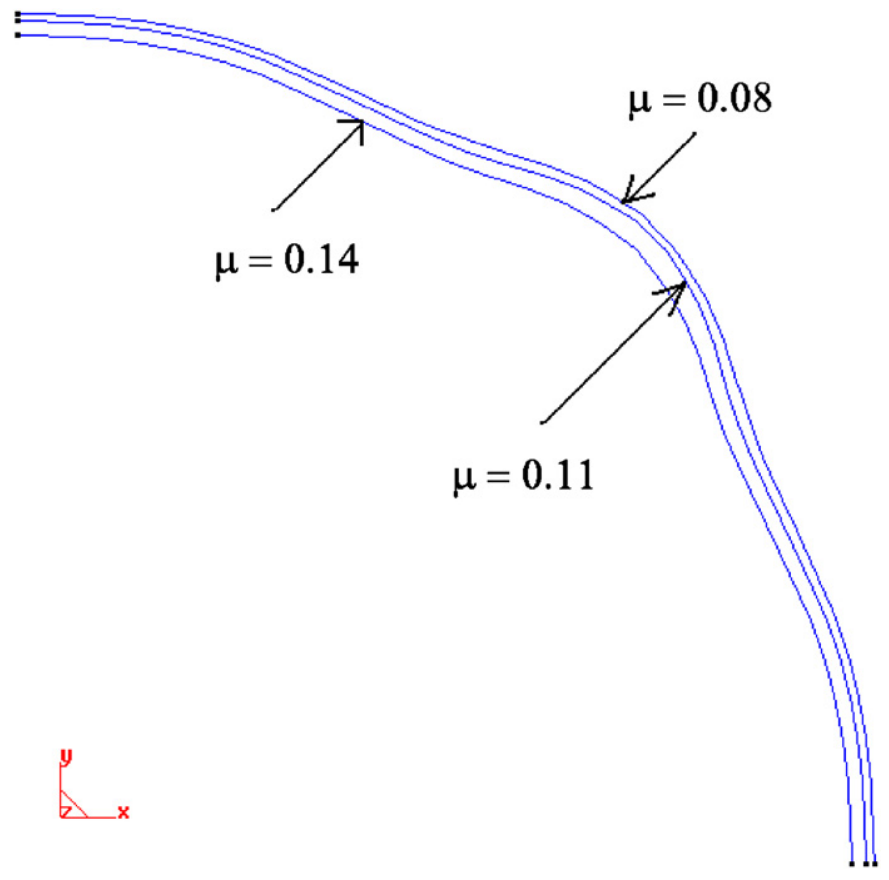

Fig. 13. Optimal blank shape using different friction coefficients.

significantly alters the optimal blank shape required for a part. Smaller blank is required for a part when the contact friction condition is high while a larger blank is necessary for a low friction condition, as shown in the figure.

\section{Conclusions}

Initial anisotropy and thus the orientation of the blank rolling direction have strong influence on the flow characteristics of the blank and in the optimal blank for a part. The blank shape optimization method described in this paper considers the initial anisotropy present in the blank and determines an optimal blank shape for any sheet metal part accordingly. It is evident from the simulation results that the described method is capable of achieving the optimal blank shape for a part within four iterations and the blank shape depends on the orientation of the sheet rolling direction with respect to an axis. The described blank shape optimization procedure accounts the deformation behaviour of blank while determining an optimal blank shape. Hence, it is concluded that optimal blank for any industrial part can be determined using the proposed method.

\section{Acknowledgements}

The authors are grateful to the Portuguese Foundation for Science and Technology (FCT) for the financial support of this work, through the Program POCI 2010.

\section{References}

[1] L.P. Moreira, G. Ferron, G. Ferran, Experimental and numerical analysis of the cup drawing test for orthotropic metal sheets, J. Mater. Process. Technol. 108 (2000) 78-86.

[2] J.W. Yoon, F. Barlat, K. Chung, F. Pourboghrat, D.Y. Yang, Earing predictions based on asymmetric nonquadratic yield function, Int. J. Plast. 16 (2000) 1075-1104.

[3] O. Engler, S. Kalz, Simulation of earing profiles from texture data by means of a visco-plastic self-consistent polycrystal plasticity approach, Mater. Sci. Eng. A 373 (2004) 350-362.
[4] M.J. Worswick, M.J. Finn, The numerical simulation of stretch flange forming, Int. J. Plast. 16 (2000) 701-720.

[5] P.D. Wu, S.R. MacEwen, D.J. Lloyd, M. Jain, P. Tugcu, K.W. Neale, On pre-straining and the evolution of material anisotropy in sheet metals, Int. J. Plast. 21 (2005) $723-739$.

[6] T. Kuwabara, W.-H. Si, PC-based blank design system for deep drawing irregularly shaped prismatics shells with arbitrarily shaped flange, J. Mater. Process. Technol. 63 (1997) 89-94.

[7] M.H. Parsa, P.H. Matin, M.M. Mashhadi, Improvement of initial blank shape for intricate products using slip line field, J. Mater. Process. Technol. 145 (2004) 21-26.

[8] S.H. Park, J.W. Yoon, D.Y. Yang, Y.H. Kim, Optimum blank design in sheet metal forming by the deformation path iteration method, Int. J. Mech. Sci. 41 (1999) 1217-1232.

[9] C.H. Lee, H. Huh, Blank design and strain estimates for sheet metal forming processes by a finite element inverse approach with initial guess of linear deformation, J. Mater. Process. Technol. 82 (1998) 145-155.

[10] Y.Q. Guo, J.L. Batoz, H. Naceur, S. Bouabdallah, F. Mercier, F. Barlat, Recent developments on the analysis and optimum design of sheet metal forming parts using a simplified inverse approach, Comput. Struct. 78 (2000) 133-148.

[11] J.-Y. Kim, N. Kim, M.-S. Huh, Optimum blank design of an automobile subframe, J. Mater. Process. Technol. 101 (2000) 31-43.

[12] L.F. Menezes, C. Teodosiu, Three-dimensional numerical simulation of the deepdrawing process using solid finite elements, J. Mater. Process. Technol. 97 (2000) 100-106.

[13] M.C. Oliveira, J.L. Alves, B.M. Chaparro, L.F. Menezes, Study on the influence of work-hardening modeling in springback prediction, Int. J. Plast. 23 (2007) 516-543.

[14] M.C. Oliveira, J.L. Alves, L.F. Menezes, Improvement of a frictional contact algorithm for strongly curved contact problems, Int. J. Numer. Methods Eng. 58 (2003) 2083-2101.

[15] A.J. Baptista, J.L. Alves, D.M. Rodrigues, L.F. Menezes, Trimming of 3D solid finite element meshes using parametric surfaces: application to sheet metal forming, Finite Elem. Anal. Des. 42 (2006) 1053-1060.

[16] L. Piegl, W. Tiller, The NURBS Book, second ed., Springer, Berlin, 1997.

[17] B. da Rocha, J. Ferreira Duarte, Tecnologia da Embutidura, Associação Portuguesa das Tecnologias de Conformação Plástica (APTCP), 1993.

[18] G. Maeder, Material forming and dimensioning problems: expectations from the car industry, in: D. Banabic (Ed.), Proceedings of 8th ESAFORM conference, The publishing house of the Romanian academy, 2005, pp. 11-20.

[19] R. Hill, A theory of the yielding and plastic flow of anisotropic metals, Proc. R. Soc. London A 193 (1948) 281-297.

[20] F. Barlat, D.J. Lege, J.C. Brem, A six component yield function for anisotropic materials, Int. J. Plast. 7 (1991) 693-712.

[21] F. Barlat, H. Aretz, J.W. Yoon, M.E. Karabin, J.C. Brem, R.E. Dick, Linear transformation-based anisotropic yield functions, Int. J. Plast. 21 (2005) 1009-1039.

[22] A.P. Karafillis, M.C. Boyce, A general anisotropic yield criterion using bounds and a transformation weighting tensor, J. Mech. Phys. Solids 41 (1993) 1859-1886.

[23] D.C. Drucker, Relation of experiments to mathematical theories of plasticity, J. Appl. Mech. 16 (1949) 349-360.

[24] Y.S. Suh, F.I. Saunders, R.H. Wagoner, Anisotropic yield functions with plasticstrain-induced anisotropy, Int. J. Plast. 12 (1996) 417-438.

[25] S. Bouvier, J.L. Alves, M.C. Oliveira, L.F. Menezes, Modelling of anisotropic workhardening behaviour of metallic materials subjected to strain-path changes, Comput. Mater. Sci. 32 (2005) 301-315.

[26] O.S. Hooperstad, T. Berstad, H. Ilstad, O.G. Lademo, M. Langseth, Effects of the yield criterion on local deformations in numerical simulation of profile forming, J. Mater. Process. Technol. 80-81 (1998) 551-555.

[27] W.F. Hosford, Reflection on the dependence of plastic anisotropy on texture, Mater. Sci. Eng. A 257 (1998) 1-8.

[28] P.D. Wu, M. Jain, J. Savoie, S.R. MacEwen, P. Tugcu, K.W. Neale, Evaluation of anisotropic yield functions for aluminum sheets, Int. J. Plast. 19 (2003) 121-138.

[29] L. Duchêne, A.M. Habraken, Analysis of the sensitivity of FEM predictions to numerical parameters in deep drawing simulations, Eur. J. Mech. A. Solids 24 (2005) 614-629.

[30] O. Cazacu, F. Barlat, Generalization of Drucker's yield criterion to orthotropy, Math. Mech. Solids 6 (2001) 613-630.

[31] B.M. Chaparro, I.L. Alves, L.F. Menezes, I.V. Fernandes, Optimization of the phenomenological constitutive models parameters using genetic algorithms, in: D. Banabic (Ed.), Advanced Methods in Material Forming, Springer, Berlin, 2007.

[32] J.L. Alves, Simulação Numérico do Processo de Stampagem Chappas de Metálicas, Ph.D. Thesis, University of Minho, Portugal, 2003.

[33] J.L. Alves, M.C. Oliveira, L.F. Menezes, An advanced constitutive model in sheet metal forming simulation: the Teodosiu microstructural model and the Cazacu Barlat yield criterion, in: S. Glosh, J.C. Castro, J.K. Lee (Eds.), Proceedings NUMIFORM'04, American Institute of Physics, Melville, E.U.A, 2004, p. 1645.

[34] J.W. Yoon, F. Barlat, R.E. Dick, M.E. Karabin, Prediction of six or eight ears in a drawn cup based on a new anisotropic yield function, Int. J. Plast. 22 (2006) 174-193. 